\title{
Intratumoral expression of CCR3 in breast cancer is associated with improved relapse-free survival in luminal-like disease
}

\author{
Di-He Gong ${ }^{1,2, *}$, Lei Fan ${ }^{1, *}$, Hai-Yan Chen ${ }^{3}$, Ke-Feng Ding ${ }^{3}$, Ke-Da Yu ${ }^{1}$ \\ ${ }^{1}$ Department of Breast Surgery, Shanghai Cancer Center and Cancer Institute, Shanghai Medical College, Fudan University, \\ Shanghai, 200032, P. R. China \\ ${ }^{2}$ Department of Thyroid and Breast Surgery, Affiliated Cixi Hospital, Wenzhou Medical University, Cixi Zhejiang, 315300, \\ P. R. China \\ ${ }^{3}$ Department of Surgical Oncology, The Second Affiliated Hospital of Zhejiang University School of Medicine, Hangzhou \\ Zhejiang, 310009, P. R. China \\ *These authors have contributed equally to this work \\ Correspondence to: Di-He Gong, e-mail: gongdihe@163.com \\ Ke-Da Yu, e-mail: yukeda@163.com \\ Keywords: CCR3, breast cancer, peritumoral, luminal-like, relapse-free survival \\ Received: December 21, $2015 \quad$ Accepted: March 28, $2016 \quad$ Published: April 11, 2016
}

\section{ABSTRACT}

Purpose: The association chemokine receptor CCR3 with breast cancer subtypes and relapse-free survival is unknown.

Results: The overall expression (either intratumoral or peritumoral) of CCR3 was not associated with tumor size, lymph node status, age, and subtype. When we confined the analysis in samples without peritumoral stromal CCR3 expression, intratumoral expression of CCR3 was associated with breast cancer subtype $(P=0.04)$. Tumors with high expression of CCR3 were more likely to be luminal-like rather than TNBC or HER2-enriched cancers. Moreover, high mRNA expression of CCR3 was related with improved relapse-free survival in luminal-A/B $(P<0.001)$. The subsequent sensitivity analysis using the systemically untreated patients confirmed that higher mRNA expression of CCR3 was a robust prognostic factor for luminal-A $(P=0.0025)$ and luminal-B $(P=0.088)$, but not for HER2-enriched $(P=0.21)$ and TNBC $(P=0.86)$. In the independent cohort, the positive association between increased expression of CCR3 and improved distant relapse-free survival was also observed.

Methods: We determined the expression level of CCR3 in 150 cases with breast cancer by using immunohistochemistry (IHC) assay, for both intratumoral and peritumoral stroma, and investigated the effect of CCR3 expression on relapse-free survival according to subtype using cases from publicly available datasets, in the whole group $(\mathrm{N}=3557)$ and in the patients without adjuvant systemic treatment $(\mathrm{N}=1005)$, respectively. Moreover, the survival outcomes were validated in another independent cohort including 508 breast cancer patients treated with neoadjuvant chemotherapy.

Conclusions: Our data indicate that intratumoral expression of CCR3 in breast cancer is associated with improved relapse-free survival in patients with luminal-like disease.

\section{INTRODUCTION}

Recent development of gene expression microarray technology has made the intrinsic molecular classification of breast cancer possible [1]. Different subtypes of breast cancer exhibit diverse behaviors, varied prognosis, as well as different response to systemic treatment [2]. Among the known four subtypes (luminal-A, luminal-B, HER2enriched, and triple-negative breast cancer [TNBC]), TNBC and HER2-enriched subtypes display more aggressive behaviors compared with luminal-like disease, which is defined by the moderate to adequate expression of estrogen receptor (ER) and/or progesterone receptor (PR). 
Cytokines are a protein family of regulatory factors derived from tumors and their environmental components that contribute to the growth, invasion, and metastasis of cancer. CCR3 is identified to be one of the major factors that involve in the progression and metastasis of some human tumors [3-5]. For instance, CCR3/eotaxin-1 loop has been revealed to increase the growth of malignant tumor cells in T-cell lymphomas [4], and the presence of CCR3 in human renal cell carcinoma samples correlates with the grade of malignancy [5]. Moreover, CCR3 and CCR10 are the known receptors for CCL28 [6], which could promote tumor tolerance and angiogenesis [7]. However, to our best knowledge, few studies have addressed the association between CCR3 and breast cancer, especially for different breast cancer subtypes. Interestingly, a previous report regarding the analysis of the expression profiles of cytokines and cytokine-related genes during the progression of breast cancer growth in mice showed that CCR3 was up-regulated in tumor tissues, but down-regulated in lymph nodes during tumor growth [8], indicating that CCR3 might be a protective factor during cancer development and metastasis.

The aim of this study was to detect the expression of CCR3 in both breast tumor and tumor stroma, and to investigate the association of CCR3 expression with different breast cancer subtypes. Furthermore, the effect of CCR3 on survival was explored in publicly available databases.

\section{PATIENTS AND METHODS}

\section{Study patients and samples}

The tumor samples used in this study were from patients with operable primary invasive breast carcinoma (stages I to IIb and T3N1M0) who received surgical therapy between May-2010 and March-2013, at the Department of Thyroid and Breast Surgery in Affiliated Cixi Hospital of Wenzhou Medical University. Tumors were staged according to the American Joint Committee on Cancer (AJCC) pathologic tumor-node-metastasis (TNM) classification (the $7^{\text {th }}$ edition). One hundred and fifty consecutive cases were included in this study and the basic information of clinical and pathological characteristics are shown in Table 1. Exclusion criteria included neoadjuvant chemotherapy, prior malignancies, and stage IV disease. All patients were female, with the median age of 52 years (ranging from 24 to 75 years). The research protocol was reviewed and approved by the Ethical Committee of the Affiliated Cixi Hospital of Wenzhou Medical University. All participants provided written informed consents.

\section{Measurement of ER, PR, and HER2 status}

Immunohistochemistry (IHC) assessment of ER, PR, and HER2 expression was conducted in paraffin-embedded tumor samples obtained from surgical operation according to American Society of Clinical Oncology/College of American Pathologists (ASCO/CAP) guidelines [9, 10]. ER and PR are considered positive if there are at least $1 \%$ positive tumor nuclei in the sample on testing in the presence of expected reactivity of internal and external controls [9]. HER2 positivity was determined by IHC $3+$ (HerceptTest, DAKO, Denmark) or fluorescence in situ hybridization (FISH) positive status (PathVysion HER2 DNA probe kit) [10]. Molecular subtype was categorized as following [11, 12]: luminal-A, ER and/or PR positive and HER2 negative; luminal-B, ER and/or PR positive and HER2 positive; HER2enriched, ER and PR negative and HER2 positive; TNBC, ER, PR, and HER2 negative. Consequently, there were 86 (57\%) luminal-A, 21 (14\%) luminal-B, 27 (18\%) TNBC, and 16 (11\%) HER2-enriched breast cancers.

\section{IHC assay for CCR3}

IHC assay of CCR3 expression was performed, for both intratumoral and peritumoral stroma. The procedure of IHC assay had been described previously [13]. In brief, four-micron paraffin sections were prepared, and tissue sections were deparaffinized in xylene for 5 minutes and rehydrated with graded ethanol. The endogenous peroxidase activity was blocked with 3\% hydrogen peroxide for 10 minutes. Antigen retrieval was done with $10 \mathrm{mM}$ citrate buffer. The sections were incubated with diluted goat serum for 10 minutes followed by incubated overnight with the primary CCR 3 rabbit monoclonal antibody (dilution 1:500, Rabbit monoclonal to CCR3, clone ab32512, Abcam Corp. Cambridge, UK) at $4^{\circ} \mathrm{C}$. The slides were incubated with biotinylated secondary antibody for 30 minutes and then the slides were incubated for 30 minutes with streptavidine-peroxidase. Staining development was performed with 3-3'-diaminobenzidine. Negative controls were carried out by replacement of the primary antibody with substituting phosphate buffer saline.

The immunereactivity was evaluated according to Hao's method [14]. Briefly, immunostaining was independently examined by two clinical pathologists who were unaware of the patient's clinico-pathologic information. For each sample, five high-power fields $(200 \times)$ were randomly selected. Cytoplasmic and membranaceous staining intensity and percentage of positive tumor cells were assessed. The extent of staining was categorized into five semi-quantitative classes based on the percentages of positive tumor cells: $0(<5 \%$ positive cells), 1 (6 to $25 \%$ positive cells), 2 (26 to $50 \%$ positive cells), 3 (51 to $75 \%$ positive cells), and 4 ( $>75 \%$ positive cells). The intensity of staining was also determined semiquantitatively on a scale of 0 to 3 as followings: 0 (negative), 1 (weak staining), 2 (moderate staining), and 3 (strong staining). Multiplication of the intensity 


\begin{tabular}{lccc}
\hline & & Number & \% \\
\hline Median age (min to max), & & 52 (24 to 85) & \\
years & & & 11.3 \\
Tumor size & $\leq 2 \mathrm{~cm}$ & 17 & 88.7 \\
& $>2 \mathrm{~cm}$ & 133 & 62.0 \\
Lymph node & Negative & 93 & 38.0 \\
& Positive & 57 & 35.3 \\
ER status & Negative & 53 & 64.7 \\
& Positive & 97 & 37.3 \\
PR status & Negative & 56 & 62.7 \\
& Positive & 94 & 75.3 \\
HER2 status & Negative & 113 & 24.7 \\
Subtype & Positive & 37 & 57.3 \\
& Luminal-A & 86 & 14.0 \\
& Luminal-B (HER2-positive) & 21 & 18.0 \\
Triple-negative & 27 & 10.7 \\
CCR3 (intratumoral & HER2-enriched & 16 & 13.3 \\
expression) & Negative & 20 & 86.7 \\
\hline
\end{tabular}

and the percentage scores gave the final staining scores [14]: 0 to 2 , as negative; 3 to 12 , as positive. Disparity of results between the two pathologists were resolved by discussion, and a consensus was reached. The representative CCR3 IHC staining plots are shown in Figure 1.

\section{Survival analysis}

Because of short follow-up time and rare event among our study cases, we could not analyze survival outcome in our cohort. Instead, we conducted the survival analysis in an online tool using the publicly available datasets. This online database named Kaplan Meier plotter was established using gene expression data and survival information of 4142 breast cancer patients (2014 version) downloaded from Gene Expression Omnibus (GEO). CCR3 gene (probe set, 208304_at) was entered into the database (http://kmplot.com/breast/, last accessing time 2015-12-20) to obtain Kaplan-Meier survival plot where the number-at-risk was indicated below the main plot [15, 16]. Hazard ratio (HR) with $95 \%$ confidence intervals
(CI) and log-rank P were calculated and displayed on the webpage. The primary endpoint of interest was relapsefree survival (RFS). We investigated the effect of CCR3 expression on RFS by breast cancer subtype, both in all the patients $(\mathrm{N}=3557)$ and in the patients without adjuvant systemic treatment $(\mathrm{N}=1005$, received neither adjuvant chemotherapy nor endocrine therapy).

\section{Validation cohort for survival outcome}

To validate the association of CCR3 expression with survival, we chose an independent cohort of patients treated with neoadjuvant chemotherapy. This cohort from M.D. Anderson Cancer Center (MDACC, Houston, TX) has been described previously [17, 18]. Briefly, these 508 patients were those with newly diagnosed HER2-negative breast cancer treated with neoadjuvant chemotherapy containing sequential anthracycline and taxane-based regimens (then underwent surgery and followed by endocrine therapy if ER-positive). All gene expression microarrays were profiled in the Department of Pathology at the MDACC and the details of the methods for RNA purification and microarray hybridization have been 
reported previously $[19,20]$. The study endpoint was distant relapse-free survival (DRFS), which was calculated from initial diagnostic biopsy of breast cancer to the occurrence of distant metastasis or non-breast cancer death. Data sets for this study are accessible via the GEO repository under accession identification numbers GSE25066 [18].

\section{Statistics}

The association between CCR3 expression level and clinico-pathologic variables was assessed by $\chi^{2}$ test. The Kaplan-Meier method was used to draw survival plot. Two-sided $\mathrm{P}<0.05$ was considered statistically significant. All of the statistical analysis was performed using SPSS 17.0 (SPSS Inc, Chicago, IL, USA).

\section{RESULTS}

\section{Association between CCR3 expression and clinico-pathologic variables}

We first evaluated the association between IHC expression level of CCR3 and clinico-pathologic characteristics in our study cohort. This analysis included 150 patients. However, we found that overall CCR3 expression (either intratumoral or peritumoral) was not associated with tumor size, lymph node status, age, and subtype (defined by ER, PR, and HER2 status)(data not shown).

\section{Intratumoral and peritumoral stromal expression of CCR3 in breast cancer by subtype}

We noticed that, in a minority of cases $(14 \%$, 21/150), peritumoral stroma displayed positive CCR3. The interaction between intratumoral and peritumoral stromal expression of CCR3 is shown in Table 2. When the peritumoral stroma was CCR3 positive, the intratumoral expression of CCR3 would be positive; however, when intratumoral expression of CCR3 was positive, only a few cases would peritumorally positive.

Since peritumorally expressed CCR3 might be a confounding factor for tumor initiation and subtype development, we therefore confined our analysis in samples without peritumoral stromal expression of CCR3. Interestingly, when peritumoral stromal CCR3 was weak or absent, intratumoral expression of CCR 3 was associated with breast cancer subtype $(\mathrm{P}=0.04)$ (Table 3$)$. Tumors with intratumoral CCR3 expression were more likely to be luminal-like rather than TNBC or HER2-enriched. Of note, HER2-enriched cases were more likely to be intratumoral CCR3 negative.

\section{Survival analysis of CCR3 according to breast cancer subtype}

Subsequently, to evaluate the prognostic effect of CCR3, we constructed Kaplan-Meier plots and log-rank analyses in 3557 patients in an online database. At first, we performed this analysis using the "best cutoff" value of mRNA of CCR3 (all percentiles are computed and the best performing threshold is automatically chosen as the cutoff). In the whole population, high CCR3 expression was an indicator of reduced risk for relapse $(\mathrm{P}<0.001)$. When we analyzed according to subtype, the high expression of CCR3 was related to improved RFS in luminal-A $(\mathrm{P}<0.001$, Figure $2 \mathrm{~A})$, luminal-B $(\mathrm{P}<0.001$, Figure 2B), HER2-enriched ( $\mathrm{P}=0.013$, Figure $2 \mathrm{C})$ cases, but not in TNBC $(\mathrm{P}=0.07$, Figure $2 \mathrm{D})$ cases. To confirm these findings, we performed a sensitivity analysis
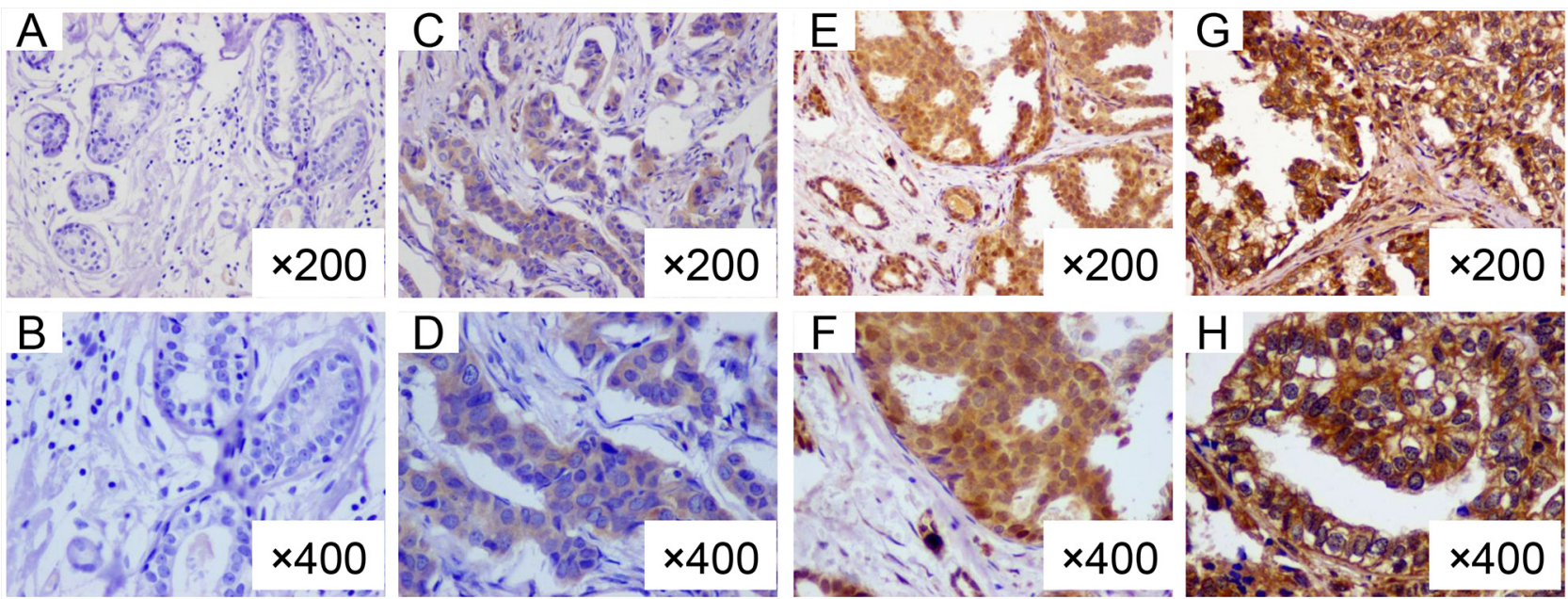

Figure 1: Representative immunohistochemical staining of CCR3 in breast cancers. A. $(\times 200)$ and B. $(\times 400)$, intratumor negative and peritumoral stroma negative for CCR3; C. $(\times 200)$ and D. $(\times 400)$, intratumor moderate positive and peritumoral stroma negative; E. $(\times 200)$ and F. $(\times 400)$, intratumor strong positive and peritumoral stroma negative; G. $(\times 200)$ and $\mathbf{H}$. $(\times 400)$, intratumor positive and peritumoral stroma positive for CCR3 
Table 2: Association between intratumoral and peritumoral stromal expression of CCR3 (N=150)

\begin{tabular}{lccccc}
\hline & \multicolumn{3}{c}{ Peritumoral stromal expression of CCR3 } & P \\
\cline { 3 - 6 } & & Negative & $\%$ & Positive & \% \\
\hline $\begin{array}{l}\text { Intratumoral } \\
\text { expression of } \\
\text { CCR3 }\end{array}$ & Negative & 20 & 15.5 & 0 & 0 \\
& Positive & 109 & 84.5 & 21 & 100 \\
\hline
\end{tabular}

*Fisher exact test $\mathrm{P}=0.07$

Table 3: Association of intratumoral expression of CCR3 with tumor size, node status, and subtype, in cases without CCR3 expression in peritumoral stroma $(\mathrm{N}=129)$

\begin{tabular}{|c|c|c|c|c|c|c|}
\hline & & \multicolumn{2}{|c|}{ Intratumoral CCR3 negative } & \multicolumn{2}{|c|}{ Intratumoral CCR3 positive } & \multirow[t]{2}{*}{$\mathbf{P}$} \\
\hline & & Number & $\%$ & Number & $\%$ & \\
\hline \multirow[t]{2}{*}{ Tumor size } & $\leq 2 \mathrm{~cm}$ & 3 & 20.0 & 12 & 80.0 & 0.61 \\
\hline & $>2 \mathrm{~cm}$ & 17 & 14.9 & 97 & 85.1 & \\
\hline \multirow[t]{2}{*}{ Lymph node } & Negative & 10 & 12.7 & 69 & 87.3 & 0.26 \\
\hline & Positive & 10 & 20.0 & 40 & 80.0 & \\
\hline \multirow[t]{4}{*}{ Subtype } & Luminal-A & 10 & 13.7 & 63 & 86.3 & 0.04 \\
\hline & $\begin{array}{c}\text { Luminal-B } \\
\text { (HER2-positive) }\end{array}$ & 1 & 5.3 & 18 & 94.7 & \\
\hline & Triple-negative & 4 & 16.0 & 21 & 84.0 & \\
\hline & HER2-enriched & 5 & 41.7 & 7 & 58.3 & \\
\hline
\end{tabular}

using the systemically untreated patients and using the median value of CCR3 mRNA as the cutoff. It showed that relatively higher expression of $\mathrm{CCR} 3$ was a robust prognostic factor for luminal-A $(\mathrm{P}=0.0025$, Figure $3 \mathrm{~A})$ and luminal- $\mathrm{B}$ (borderline $\mathrm{P}=0.088$, Figure $3 \mathrm{~B}$ ), but not for HER2-enriched $(\mathrm{P}=0.21$, Figure $3 \mathrm{C})$ and TNBC $(\mathrm{P}=0.86$, Figure 3D).

\section{Validation for survival outcome}

We chose the M.D. Anderson cohort for validation. The Kernel density estimate method was used to determine the optimal cutoff of CCR3 mRNA level (Figure 4A). Using the expression of 6.0 as the cutoff value, we analyzed DRFS according to PAM50-predicted subtypes. The higher expression of CCR3 was related to improved DRFS in luminal-like disease $(\mathrm{P}=0.068$, Figure 4B left), but not in TNBC $(\mathrm{P}=0.62$, Figure $4 \mathrm{~B}$ right $)$ or normal-like disease $(\mathrm{P}=0.82$, plot not shown).

\section{DISCUSSION}

In the present study, we found that the intratumoral expression of CCR3 was associated with luminal- like disease. Moreover, we demonstrated that higher intratumoral mRNA expression of CCR3 reduced the risk for cancer relapse in luminal-like disease but not in TNBC and HER2-enriched cancers.

Recent findings have suggested chemokines and their receptors play important roles in cancer biology by influencing the tumor microenvironment and regulating cancer cell function [21]. CCR3 is a novel receptor associated with development, progression, and aggressiveness of several types of cancer. In the present study, we examined its expression in luminal-A/B, TNBC, and HER2-enriched subtypes, and showed that the expression of CCR3 is universal $(87 \%$ intratumoral expression). Of note, high CCR3 expression was more prevalent in luminal-like cases, indicating CCR3 might be involved in luminal-like cancer development. In contrast, CCR3 positive rate was relatively low in TNBC or HER2-enriched cancers, implied that the diverse effect of CCR3 in development of different breast cancer subtypes. Previous research also suggested that CCL5/ CCR3 signaling pathway is involved in prognosis and immunotherapy of luminal breast cancer [22]. To the best of our knowledge, the current study represents the first comprehensive investigation reporting the positive 
association between CCR3 expression and luminal-like breast cancer. It warrants further investigation regarding the precise mechanism of CCR3 modulating luminal-like cancer.

Furthermore, higher expression of CCR3 is significantly associated with improved RFS in luminal-A and luminal-B cases, suggesting that CCR3 may reduce or delay metastases in breast cancer. We not only found the protective effect of CCR3 in unselected population, but also successfully repeated the outcomes in patients without adjuvant systemic treatment, indicating a robust and steady effect of CCR3 on breast cancer relapse. To validate the findings, we chose an independent cohort of 508 patients treated with neoadjuvant chemotherapy from MDACC. Again, higher expression of CCR3 was related to improved DRFS in luminal-like disease but not in TNBC or normal-like disease. Our study had some limitation. First, the association between intratumoral expression of CCR3 and luminal-like disease is only observed in our cohort of 150 cases, and needs further validation. Second, we measured the protein (by IHC) of intratumoral and peritumoral stromal expression of CCR3 in subtype association analysis while investigated the mRNA of CCR3 in survival analysis. There might be inconsistent between protein and mRNA. The last but not the least, despite of the large number of sample size and successful validation in the survival analysis, we must point out that the publicly available gene microarray databases from different institutions and laboratories could inevitably introduce bias.

In conclusion, we have shown for the first time that the higher mRNA expression of CCR3 indicates a decreased risk of relapse in luminal-A and luminal-B cases, but not in TNBC and HER2-enriched patients. The precise mechanism underlying the association between CCR3 and luminal-like disease deserves further investigation.
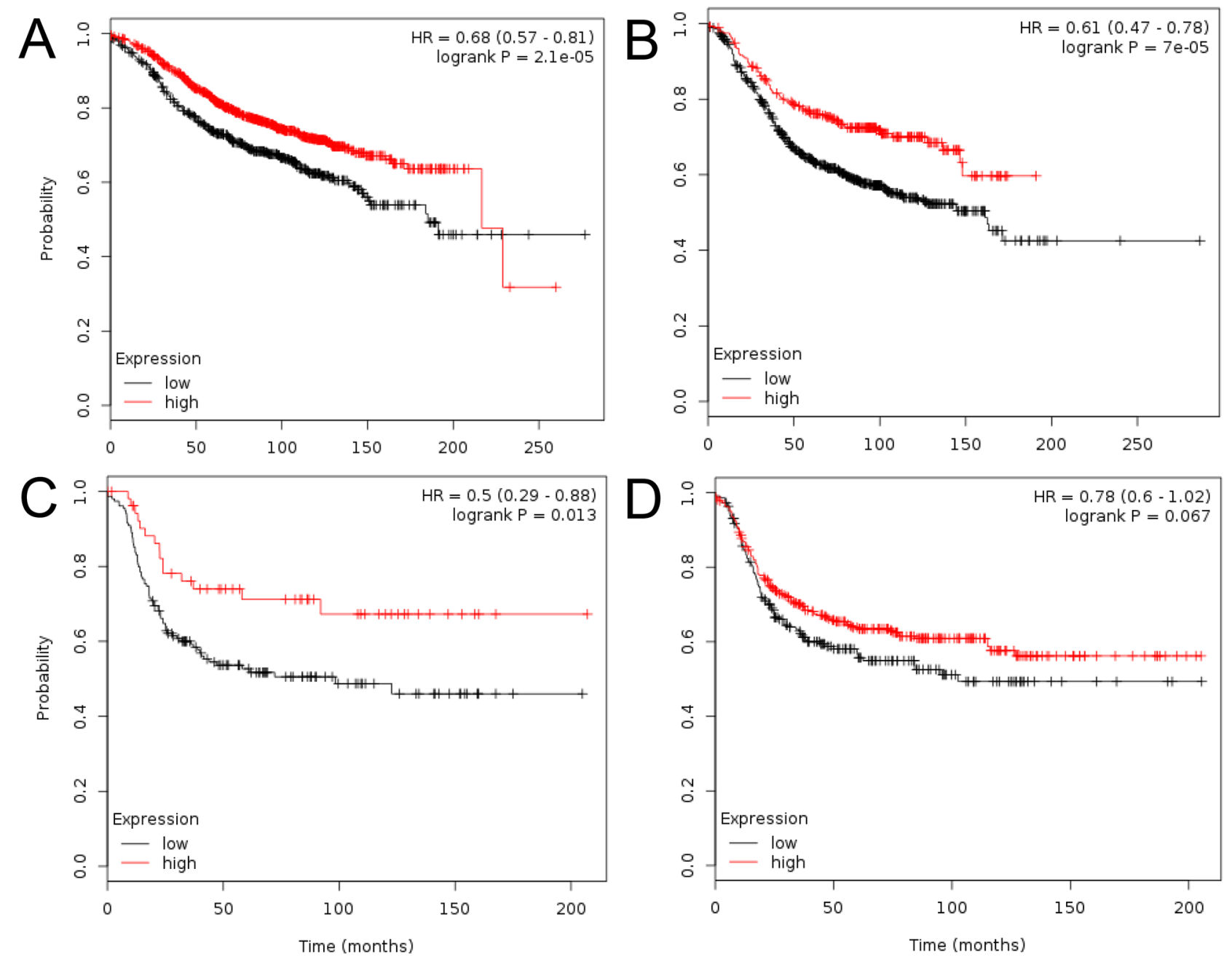

Figure 2: Kaplan-Meier plots derived from http://kmplot.com/analysis/. The analyses were performed and the Kaplan-Meier plots were drawn regarding CCR3 expression and relapse-free survival in the luminal-A A. luminal-B B. triple-negative C. and HER2enriched D. The "best cutoff" for CCR3 mRNA was used. 

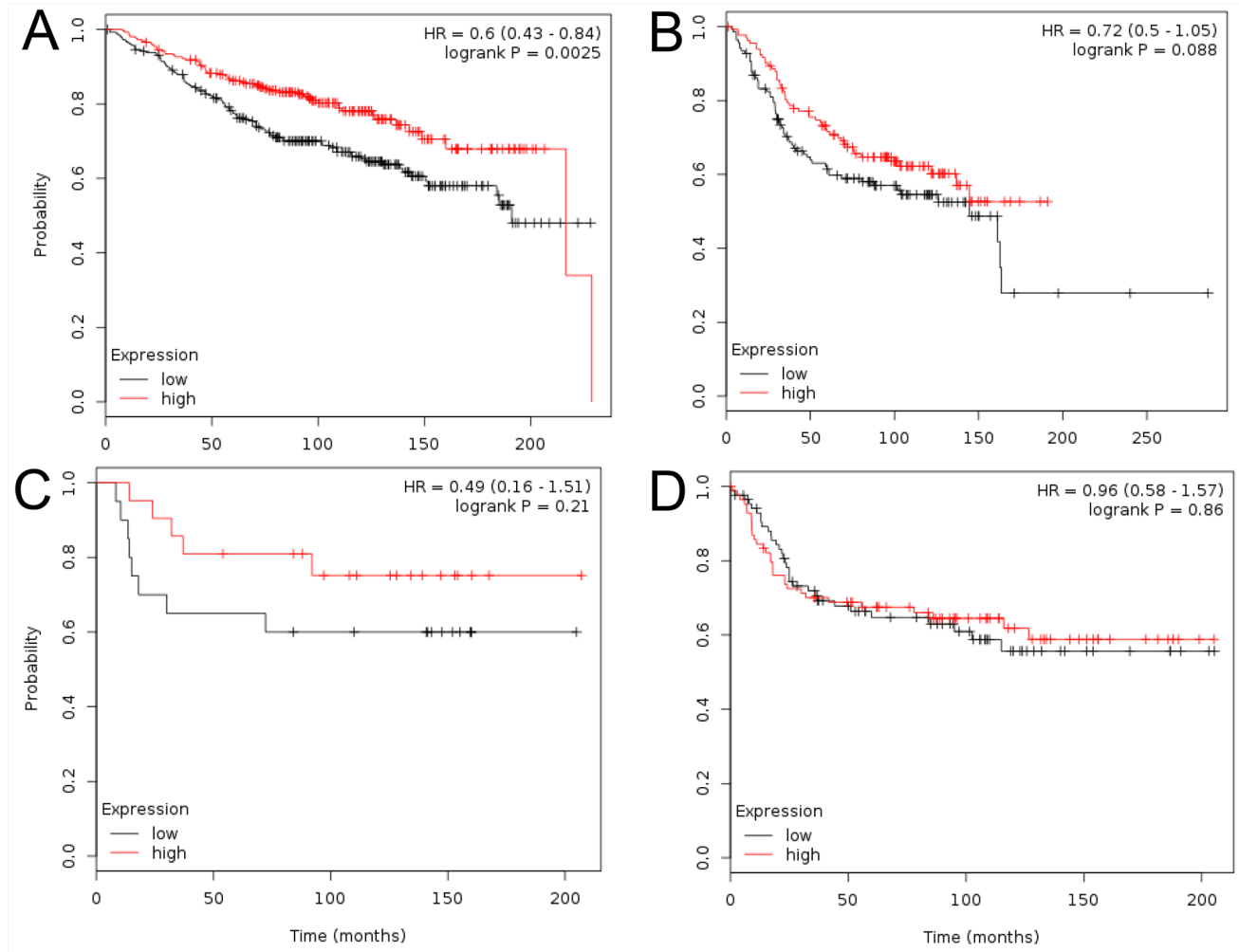

Figure 3: Kaplan-Meier plots in systemically untreated patients. The patients were split by median expression of CCR3 mRNA. Plots are shown according to subtype: luminal-A A. luminal-B B. triple-negative C. and HER2-enriched D.

A

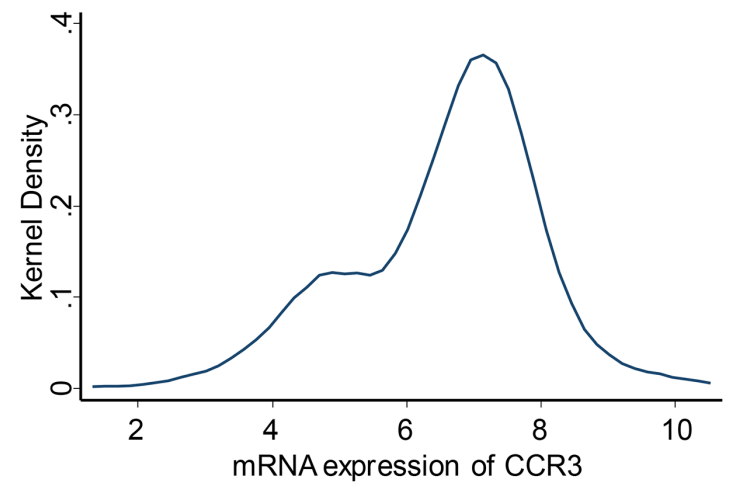

B
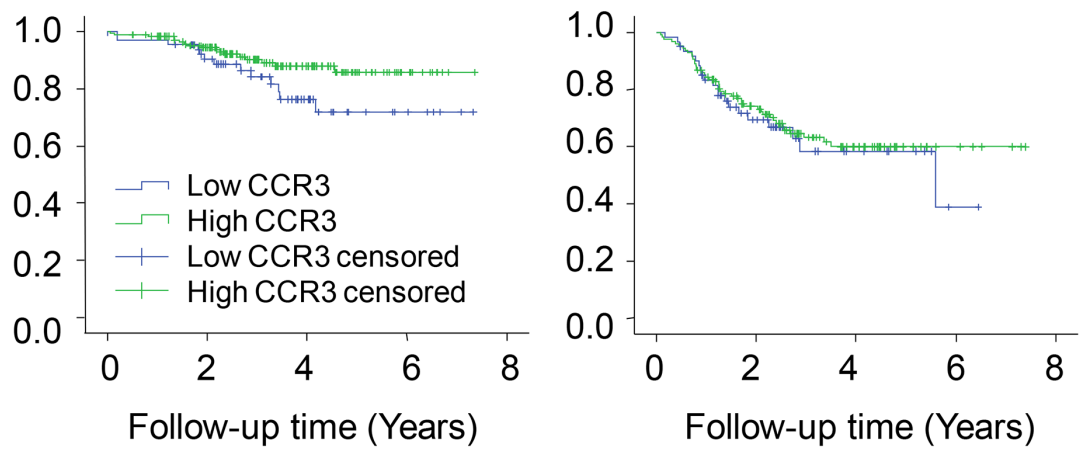

Figure 4: Validating the effect of CCR3 expression on distant relapse-free survival (DRFS) in patients receiving neoadjuvant chemotherapy. The Kernel density estimate was used to determine the optimal cutoff of CCR3 mRNA level A. KaplanMeier plots are shown according to subtype: luminal-like B, left, and triple-negative $\mathbf{B}$, right. 


\section{ACKNOWLEDGEMENT}

We thank the patients for their willingness to cooperate with our study. The National Natural Science Foundation of China (81001169) and the Training Plan of Excellent Talents in Shanghai Municipality Health System (XYQ2013101) supported this work. The funders had no role in the study design, data collection and analysis, decision to publish, or preparation of the manuscript.

\section{CONFLICTS OF INTEREST}

The authors declare no conflicts of interests.

\section{REFERENCES}

1. Perou CM, Sorlie T, Eisen MB, van de Rijn M, Jeffrey SS, Rees CA, Pollack JR, Ross DT, Johnsen H, Akslen LA, Fluge O, Pergamenschikov A, Williams C, Zhu SX, Lonning PE, Borresen-Dale AL, et al. Molecular portraits of human breast tumours. Nature. 2000; 406:747-752.

2. Sorlie T, Perou CM, Tibshirani R, Aas T, Geisler S, Johnsen $\mathrm{H}$, Hastie T, Eisen MB, van de Rijn M, Jeffrey SS, Thorsen T, Quist H, Matese JC, Brown PO, Botstein D, Lonning $\mathrm{PE}$, et al. Gene expression patterns of breast carcinomas distinguish tumor subclasses with clinical implications. Proc Natl Acad Sci U S A. 2001; 98:10869-10874.

3. Miyagaki T, Sugaya M, Murakami T, Asano Y, Tada Y, Kadono T, Okochi H, Tamaki K and Sato S. CCL11CCR3 interactions promote survival of anaplastic large cell lymphoma cells via ERK1/2 activation. Cancer Res. 2011; 71:2056-2065.

4. Miyagaki T, Sugaya M, Fujita H, Ohmatsu H, Kakinuma T, Kadono T, Tamaki K and Sato S. Eotaxins and CCR3 interaction regulates the Th2 environment of cutaneous T-cell lymphoma. J Invest Dermatol. 2010; 130:2304-2311.

5. Johrer K, Zelle-Rieser C, Perathoner A, Moser P, Hager M, Ramoner R, Gander H, Holtl L, Bartsch G, Greil R and Thurnher M. Up-regulation of functional chemokine receptor CCR3 in human renal cell carcinoma. Clin Cancer Res. 2005; 11:2459-2465.

6. Pan J, Kunkel EJ, Gosslar U, Lazarus N, Langdon P, Broadwell K, Vierra MA, Genovese MC, Butcher EC and Soler D. A novel chemokine ligand for CCR10 and CCR3 expressed by epithelial cells in mucosal tissues. J Immunol. 2000; 165:2943-2949.

7. Facciabene A, Peng X, Hagemann IS, Balint K, Barchetti A, Wang LP, Gimotty PA, Gilks CB, Lal P, Zhang L and Coukos G. Tumour hypoxia promotes tolerance and angiogenesis via CCL28 and T(reg) cells. Nature. 2011; 475:226-230.

8. Jung MY, Kim SH, Cho D and Kim TS. Analysis of the expression profiles of cytokines and cytokine-related genes during the progression of breast cancer growth in mice. Oncol Rep. 2009; 22:1141-1147.
9. Hammond ME, Hayes DF, Dowsett M, Allred DC, Hagerty KL, Badve S, Fitzgibbons PL, Francis G, Goldstein NS, Hayes M, Hicks DG, Lester S, Love R, Mangu PB, McShane L, Miller K, et al. American Society of Clinical Oncology/College Of American Pathologists guideline recommendations for immunohistochemical testing of estrogen and progesterone receptors in breast cancer. J Clin Oncol. 2010; 28:2784-2795.

10. Wolff AC, Hammond ME, Schwartz JN, Hagerty KL, Allred DC, Cote RJ, Dowsett M, Fitzgibbons PL, Hanna WM, Langer A, McShane LM, Paik S, Pegram MD, Perez EA, Press MF, Rhodes A, et al. American Society of Clinical Oncology/College of American Pathologists guideline recommendations for human epidermal growth factor receptor 2 testing in breast cancer. J Clin Oncol. 2007; 25:118-145.

11. Chen XS, Nie XQ, Chen CM, Wu JY, Wu J, Lu JS, Shao ZM, Shen ZZ and Shen KW. Weekly paclitaxel plus carboplatin is an effective nonanthracycline-containing regimen as neoadjuvant chemotherapy for breast cancer. Ann Oncol. 2010; 21:961-967.

12. Chen DN, Song CG, Yu KD, Jiang YZ, Ye FG and Shao ZM. A Prospective Evaluation of the Association between a Single Nucleotide Polymorphism rs3775291 in Toll-Like Receptor 3 and Breast Cancer Relapse. PLoS One. 2015; 10:e0133184.

13. Yu KD, Wang X, Yang C, Zeng XH and Shao ZM. Host genotype and tumor phenotype of chemokine decoy receptors integrally affect breast cancer relapse. Oncotarget. 2015; 6:26519-26527. doi: 10.18632/oncotarget.4470.

14. Hao L, Zhang C, Qiu Y, Wang L, Luo Y, Jin M, Zhang Y, Guo TB and Matsushima K. Recombination of CXCR4, VEGF, and MMP-9 predicting lymph node metastasis in human breast cancer. Cancer Lett. 2007; 253:34-42.

15. Gyorffy B, Lanczky A, Eklund AC, Denkert C, Budczies J, Li Q and Szallasi Z. An online survival analysis tool to rapidly assess the effect of 22,277 genes on breast cancer prognosis using microarray data of 1,809 patients. Breast Cancer Res Treat. 2010; 123:725-731.

16. Gyorffy B, Surowiak P, Budczies J and Lanczky A. Online survival analysis software to assess the prognostic value of biomarkers using transcriptomic data in non-small-cell lung cancer. PLoS One. 2013; 8:e82241.

17. Yu KD, Zhu R, Zhan M, Rodriguez AA, Yang W, Wong S, Makris A, Lehmann BD, Chen X, Mayer I, Pietenpol JA, Shao ZM, Symmans WF and Chang JC. Identification of prognosis-relevant subgroups in patients with chemoresistant triple-negative breast cancer. Clin Cancer Res. 2013; 19:2723-2733.

18. Hatzis C, Pusztai L, Valero V, Booser DJ, Esserman L, Lluch A, Vidaurre T, Holmes F, Souchon E, Wang H, Martin M, Cotrina J, Gomez H, Hubbard R, Chacon JI, Ferrer-Lozano J, et al. A genomic predictor of response and survival following taxane-anthracycline chemotherapy for invasive breast cancer. JAMA. 2011; 305:1873-1881. 
19. Symmans WF, Hatzis C, Sotiriou C, Andre F, Peintinger F, Regitnig P, Daxenbichler G, Desmedt C, Domont J, Marth C, Delaloge S, Bauernhofer T, Valero V, Booser DJ, Hortobagyi GN and Pusztai L. Genomic index of sensitivity to endocrine therapy for breast cancer. J Clin Oncol. 2010; 28:4111-4119.

20. Hess KR, Anderson K, Symmans WF, Valero V, Ibrahim N, Mejia JA, Booser D, Theriault RL, Buzdar AU, Dempsey PJ, Rouzier R, Sneige N, Ross JS, Vidaurre T, Gomez HL, Hortobagyi GN, et al. Pharmacogenomic predictor of sensitivity to preoperative chemotherapy with paclitaxel and fluorouracil, doxorubicin, and cyclophosphamide in breast cancer. J Clin Oncol. 2006; 24:4236-4244.

21. Balkwill FR. The chemokine system and cancer. J Pathol. 2012; 226:148-157.

22. Zhang Q, Qin J, Zhong L, Gong L, Zhang B, Zhang Y and Gao WQ. CCL5-Mediated Th2 Immune Polarization Promotes Metastasis in Luminal Breast Cancer. Cancer Res. 2015; 75:4312-4321. 